\title{
The Leprosy Eradication Programme of Malta
}

\author{
G. DEPASQUALE \\ St Luke's Hospital, Dept. of Dermatology, La Valetta, Malta
}

\begin{abstract}
An eradication programme ideally requires a closed community with close collaboration from all authorities concerned in order to enable the examination of all known, registered cases; early detection of cases and starting therapy with an effective, quick-acting medication to reduce the possibility of dissemination of the disease; and the facility to follow-up the patients on a long term basis. These requirements having been satisfied in the Malta Programme, one has to carry on with observation of all patients for the next few years in order to evaluate fully the results achieved.
\end{abstract}

In July 1972 a Leprosy Eradication Programme was started in the Maltese Islands with the cooperation of the Sovereign Military Order of Malta, the German Leprosy Relief Association, the Borstel Research Institute and the Ministry of Health.

The eradication programme is based on experimental and clinical studies resulting in a therapeutical method which, at least at present, can be viewed as the most effective one. It consists of a combination of rifampicin and Isoprodian (Prothionamide + isoniazid + DDS). Since the medication is administered entirely orally, and is as a general rule well tolerated, the programme could be started and carried out on an out-patient basis.

Until recent years, DDS has been the only available drug in the treatment of leprosy, from a practical point of view. Like other anti-leprosy drugs used so far, DDS is a bacteriostatic agent, limiting the reproduction of bacteria rather than killing them. This has been the rationale of lifelong therapy in dealing with leprosy.

The situation has changed with the introduction of rifampicin and the discovery of suitable combination partners. Thus a bactericidal combination enables a quicker and more intensive therapeutic effect. Treatment need no longer be a lifelong process but can be terminated in accordance with the clinical and bacteriological results of the individual case. These were the scientific prerequisites for conducting an eradication programme, which naturally differs in purpose and method from a general control programme.' On termination of therapy, subsequent observation and control of all cases has to be carried out, thus allowing a fuller evaluation of the results.

The question of control of patients, which is of such great importance in an eradication programme, proved to be no problem in the Maltese Islands. The population of c. 32,000 is fairly stable with very minimal movements. The patients have been very cooperative and regular in attendance. Patients normally 
attend once every 2 weeks at the Department of Venereology and Dermatology, St. Luke's Hospital, where they are examined and given the medicaments. Patients in Gozo, the sister island, and St. Bartholomew Hospital, are visited weekly. Home visits, when necessary, are no great difficulty due to the short distances. Patients are only admitted to hospital if suffering from a severe reaction or some complicating illness: these occasions were infrequent. Most reactions that occurred receded spontaneously or with the administration of thalidomide: steroids were never deemed necessary.

Skin smears and biopsies are taken every month from each patient. The smears and biopsies are examined at the Borstel Research Institute, while smears are also examined in Malta. An evaluation system is used integrating the smear results with those of homogenised samples and histological sections of biopsies.

When the programme was started in the second half of 1972, a total of 217 cases were registered. Subsequent adjustment of the registration list for different reasons (dubious diagnosis, movement, death, concomitant disease e.g. carcinoma, heart disease, mental disease, etc.) left 195 cases for consideration. Due to intolerance phenomena or outright refusal of treatment, 3 patients appeared to be untreatable. The remaining 192 patients form the first part of the programme. Eighteen other patients presented themselves when the project was already under way, and form an additional part of the programme.

In conformity with the character of the eradication programme, all patients were treated with the same medication, irrespective of severity of the disease, type and duration of pretreatment, age, sex, etc. Likewise, all patients were subjected to the same bacteriological evaluation system referred to above.

Of the 192 patients forming part 1 of the programme: 64 cases (34\%) were found to be "Negative", that is to say, that at no stage have their smears and biopsies exhibited any bacteria or acid-fast material. One hundred and twenty-eight cases $(66 \%)$ were found to be "Positive". In the group of 64 negative cases, treatment has been discontinued and the patients are observed every 3 months for negativity and the absence of relapse.

Of the 128 positive cases, 108 patients have reached bacteriological negativity in the sense of tissue clearance through treatment. Treatment has been discontinued in all these cases and they too are observed every 3 months for negativity and the absence of relapse. Twenty patients have not reached complete negativity in the sense of tissue clearance, but both the Bacteriological Indices and the Morphological Indices are greatly reduced. The steady decrease in the number of germs is not only demonstrated by the absolute numbers and the indices but also by the fact that the monthly controls have quite often revealed negativity in the sense of tissue clearance. These patients remain under treatment for a further period of time. Some of the patients in this group showing unsatisfactory results might be failing to take the medication regularly. On the other hand, one cannot exclude the fact that, as is seen in other diseases, some patients fail to show satisfactory results despite regular intake of the medication. A modification of therapy may have to be considered for these patients.

The group of 18 cases forming the second part of the programme, although being a heterogenous group due to the staggered initiation of treatment, enables us to make certain interesting observations. The group is composed of 5 long-standing cases that had been treated regularly or otherwise, and 13 fresh cases. Fresh cases show, as a rule, a much quicker response to therapy and the B.I. and M.I. decrease more rapidly. Clinical improvement, although related to the 
decrease in the number of bacteria, usually precedes bacterial negativation. The organism requires a relatively long time span to eliminate the acid-fast material. Its duration is not exclusively but decisively dependent upon the number of germs at the initiation of therapy. 